\title{
TENSOR PRODUCTS OF LEAVITT PATH ALGEBRAS
}

\author{
PERE ARA AND GUILLERMO CORTIÑNS \\ (Communicated by Birge Huisgen-Zimmermann)
}

\begin{abstract}
We compute the Hochschild homology of Leavitt path algebras over a field $k$. As an application, we show that $L_{2}$ and $L_{2} \otimes L_{2}$ have different Hochschild homologies, and so they are not Morita equivalent; in particular, they are not isomorphic. Similarly, $L_{\infty}$ and $L_{\infty} \otimes L_{\infty}$ are distinguished by their Hochschild homologies, and so they are not Morita equivalent either. By contrast, we show that $K$-theory cannot distinguish these algebras; we have $K_{*}\left(L_{2}\right)=K_{*}\left(L_{2} \otimes L_{2}\right)=0$ and $K_{*}\left(L_{\infty}\right)=K_{*}\left(L_{\infty} \otimes L_{\infty}\right)=K_{*}(k)$.
\end{abstract}

\section{INTRODUCTION}

Elliott's theorem 21] states that $\mathcal{O}_{2} \otimes \mathcal{O}_{2} \cong \mathcal{O}_{2}$ plays an important role in the proof of the celebrated classification theorem of Kirchberg algebras in the UCT class, due to Kirchberg [14] and Phillips [19]. Recall that a Kirchberg algebra is a purely infinite, simple, nuclear and separable $\mathrm{C}^{*}$-algebra. The Kirchberg-Phillips theorem states that this class of simple $\mathrm{C}^{*}$-algebras is completely classified by its topological $K$-theory. The analogous question whether the algebras $L_{2}$ and $L_{2} \otimes L_{2}$ are isomorphic has remained open for some time. Here $L_{2}$ is the Leavitt algebra of type $(1,2)$ over a field $k$ (see [17), that is, the $k$-algebra with generators $x_{1}, x_{2}, x_{1}^{*}, x_{2}^{*}$ and relations given by $x_{i}^{*} x_{j}=\delta_{i, j}$ and $\sum_{i=1}^{2} x_{i} x_{i}^{*}=1$.

In this paper we obtain a negative answer to this question. Indeed, we analyze a much larger class of algebras, namely the tensor products of Leavitt path algebras of finite quivers in terms of their Hochschild homology, and we prove that, for $1 \leq n<m \leq \infty$, the tensor products $E=\bigotimes_{i=1}^{n} L\left(E_{i}\right)$ and $F=\bigotimes_{j=1}^{m} L\left(F_{j}\right)$ of Leavitt path algebras of non-acyclic finite quivers $E_{i}, F_{j}$ are distinguished by their Hochschild homologies (Theorem 5.1). Because Hochschild homology is Morita invariant, we conclude that $E$ and $F$ are not Morita equivalent for $n<m$. Since $L_{2}$ is the Leavitt path algebra of the graph with one vertex and two arrows, we obtain that $L_{2} \otimes L_{2}$ and $L_{2}$ are not Morita equivalent; in particular, they are not isomorphic.

Recall that, by a theorem of Kirchberg 15, a simple, nuclear and separable $C^{*}$-algebra $A$ is purely infinite if and only if $A \otimes \mathcal{O}_{\infty} \cong A$. We also show that the analogue of Kirchberg's result is not true for Leavitt algebras. We prove in

Received by the editors August 1, 2011 and, in revised form, November 9, 2011.

2010 Mathematics Subject Classification. Primary 16E40, 16S99; Secondary 19D50.

The first author was partially supported by DGI MICIIN-FEDER MTM2008-06201-C02-01 and by the Comissionat per Universitats i Recerca de la Generalitat de Catalunya.

The second author was supported by CONICET and partially supported by grants PIP 112200801-00900, UBACyTs X051 and 20020100100386, and MTM2007-64074. 
Proposition 5.3 that if $E$ is a non-acyclic quiver, then $L_{\infty} \otimes L(E)$ and $L(E)$ are not Morita equivalent, and also that $L_{\infty} \otimes L_{\infty}$ and $L_{\infty}$ are not Morita equivalent.

Using the results in [5] we prove that the algebras $L_{2}$ and $L_{2} \otimes L(F)$, for $F$ an arbitrary finite quiver, have trivial $K$-theory: all algebraic $K$-theory groups $K_{i}$, $i \in \mathbb{Z}$, vanish on them (this follows from Lemma 6.1 and Proposition 6.2). We also compute $K_{*}(L(F))=K_{*}\left(L_{\infty} \otimes L(F)\right)$ and that $K_{*}\left(L_{\infty}\right)=K_{*}\left(L_{\infty} \otimes L_{\infty}\right)=K_{*}(k)$ is the $K$-theory of the ground field (see Proposition 6.3 and Corollary 6.4). This implies in particular that, in contrast with the analytic situation, no classification result, in terms solely of $K$-theory, can be expected for a class of central, simple $k$ algebras, containing all purely infinite simple unital Leavitt path algebras and closed under tensor products. It is worth mentioning that an important step towards a $K$-theoretic classification of purely infinite simple Leavitt path algebras of finite quivers has been achieved in 2].

We refer the reader to [3, 7] and 20 for the basics on Leavitt algebras, Leavitt path algebras and graph $\mathrm{C}^{*}$-algebras, and to 22 for a nice survey on the KirchbergPhillips Theorem.

Notation. We fix a field $k$; all vector spaces, tensor products and algebras are over $k$. If $R$ and $S$ are unital $k$-algebras, then by an $(R, S)$-bimodule we understand a left module over $R \otimes S^{o p}$. By an $R$-bimodule we shall mean an $(R, R)$ bimodule, that is, a left module over the enveloping algebra $R^{e}=R \otimes R^{o p}$. Hochschild homology of $k$-algebras is always taken over $k$. If $M$ is an $R$-bimodule, we write

$$
H H_{n}(R, M)=\operatorname{Tor}_{n}^{R^{e}}(R, M)
$$

for the Hochschild homology of $R$ with coefficients in $M$ and we abbreviate $H H_{n}(R)$ $=H H_{n}(R, R)$.

\section{HoChSCHILD HOMOLOGY}

Let $k$ be a field, $R$ a $k$-algebra and $M$ an $R$-bimodule. The Hochschild homology $H H_{*}(R, M)$ of $R$ with coefficients in $M$ was defined in the introduction. It is computed by the Hochschild complex $H H(R, M)$, which is given in degree $n$ by

$$
H H(R, M)_{n}=M \otimes R^{\otimes n} .
$$

It is equipped with the Hochschild boundary map $b$ defined by

$b\left(a_{0} \otimes a_{1} \otimes \cdots \otimes a_{n}\right)=\sum_{i=0}^{n-1}(-1)^{i} a_{0} \otimes \cdots \otimes a_{i} a_{i+1} \otimes \cdots \otimes a_{n}+(-1)^{n} a_{n} a_{0} \otimes \cdots \otimes a_{n-1}$.

If $R$ and $M$ happen to be $\mathbb{Z}$-graded, then $H H(R, M)$ splits into a direct sum of subcomplexes

$$
H H(R, M)=\bigoplus_{m \in \mathbb{Z}}{ }_{m} H H(R, M) .
$$

The homogeneous component of degree $m$ of $H H(R, M)_{n}$ is the linear subspace of $H H(R, M)_{n}$ generated by all elementary tensors $a_{0} \otimes \cdots \otimes a_{n}$ with $a_{i}$ homogeneous and $\sum_{i}\left|a_{i}\right|=m$. One of the first basic properties of the Hochschild complex is that it commutes with filtering colimits. Thus we have

Lemma 2.1. Let I be a filtered ordered set and let $\left\{\left(R_{i}, M_{i}\right): i \in I\right\}$ be a directed system of pairs $\left(R_{i}, M_{i}\right)$ consisting of an algebra $R_{i}$ and an $R_{i}$-bimodule $M_{i}$, with algebra maps $R_{i} \rightarrow R_{j}$ and $R_{i}$-bimodule maps $M_{i} \rightarrow M_{j}$ for each $i \leq j$. Let $(R, M)=\operatorname{colim}_{i}\left(R_{i}, M_{i}\right)$. Then $H H_{n}(R, M)=\operatorname{colim}_{i} H H_{n}\left(R_{i}, M_{i}\right)(n \geq 0)$. 
Let $R_{i}$ be a $k$-algebra and $M_{i}$ an $R_{i}$-bimodule $(i=1,2)$. The Künneth formula establishes a natural isomorphism ([23, 9.4.1])

$$
H H_{n}\left(R_{1} \otimes R_{2}, M_{1} \otimes M_{2}\right) \cong \bigoplus_{p=0}^{n} H H_{p}\left(R_{1}, M_{1}\right) \otimes H H_{n-p}\left(R_{2}, M_{2}\right) .
$$

Another fundamental fact about Hochschild homology which we shall need is Morita invariance. Let $R$ and $S$ be Morita equivalent algebras, and let $P \in R \otimes S^{o p}$-mod and $Q \in S \otimes R^{o p}$-mod implement the Morita equivalence. Then ([23, Thm. 9.5.6])

$$
H H_{n}(R, M)=H H_{n}\left(S, Q \otimes_{R} M \otimes_{R} P\right) .
$$

Lemma 2.3. Let $R_{1}, \ldots, R_{n}$ and $S_{1}, \ldots, S_{m}, \ldots$ be a finite and an infinite sequence of algebras, and let $R=\bigotimes_{i=1}^{n} R_{i}, S_{\leq m}=\bigotimes_{j=1}^{m} S_{j}$ and $S=\bigotimes_{j=1}^{\infty} S_{j}$. Assume:

(1) $H H_{q}\left(R_{i}\right) \neq 0 \neq H H_{q}\left(S_{j}\right) \quad(q=0,1),(1 \leq i \leq n),(1 \leq j)$.

(2) $H H_{p}\left(R_{i}\right)=H H_{p}\left(S_{j}\right)=0$ for $p \geq 2,1 \leq i \leq n, 1 \leq j$.

(3) $n \neq m$.

Then no two of $R, S_{\leq m}$ and $S$ are Morita equivalent.

Proof. By the Künneth formula, we have

$$
H H_{n}(R)=\bigotimes_{i=1}^{n} H H_{1}\left(R_{i}\right) \neq 0, \quad H H_{p}(R)=0, \quad p>n .
$$

By the same argument, $H H_{p}\left(S_{\leq m}\right)$ is non-zero for $p=m$ and zero for $p>m$. Hence if $n \neq m, R$ and $S_{\leq m}$ do not have the same Hochschild homology, and therefore they cannot be Morita equivalent, by (2.2). Similarly, by Lemma 2.1, we have

$$
H H_{n}(S)=\bigoplus_{J \subset \mathbb{N},|J|=n}\left(\bigotimes_{j \in J} H H_{1}\left(S_{j}\right)\right) \otimes\left(\bigotimes_{j \notin J} H H_{0}\left(S_{j}\right)\right)
$$

so that $H H_{n}(S)$ is non-zero for all $n \geq 1$, and thus it cannot be Morita equivalent to either $R$ or $S_{\leq m}$.

\section{Hochschild homology of CROSSED PROduCts}

Let $R$ be a unital algebra and $G$ a group acting on $R$ by algebra automorphisms. Form the crossed-product algebra $S=R \rtimes G$, and consider the Hochschild complex $H H(S)$. For each conjugacy class $\xi$ of $G$, the graded submodule $H H^{\xi}(S) \subset H H(S)$ generated in degree $n$ by the elementary tensors $a_{0} \rtimes g_{0} \otimes \cdots \otimes a_{n} \rtimes g_{n}$ with $g_{0} \cdots g_{n} \in$ $\xi$ is a subcomplex, and we have a direct sum decomposition $H H(S)=\bigoplus_{\xi} H H^{\xi}(S)$. The following theorem of Lorenz describes the complex $H H^{\xi}(S)$ corresponding to the conjugacy class $\xi=[g]$ of an element $g \in G$ as hyperhomology over the centralizer subgroup $Z_{g} \subset G$.

Theorem 3.1 ([16]). Let $R$ be a unital k-algebra, $G$ a group acting on $R$ by automorphisms, $g \in G$ and $Z_{g} \subset G$ the centralizer subgoup. Let $S=R \rtimes G$ be the crossed product algebra and $H H^{\langle g\rangle}(S) \subset H H(S)$ be the subcomplex described above. Consider the $R$-submodule $S_{g}=R \rtimes g \subset S$. Then there is a quasi-isomorphism

$$
H H^{[g]}(S) \stackrel{\sim}{\rightarrow} \mathbb{H}\left(Z_{g}, H H\left(R, S_{g}\right)\right) .
$$

In particular, we have a spectral sequence

$$
E_{p, q}^{2}=H_{p}\left(Z_{g}, H H_{q}\left(R, S_{g}\right)\right) \Rightarrow H H_{p+q}^{[g]}(S) .
$$


Remark 3.2. Lorenz formulates his result in terms of the spectral sequence alone, but his proof shows that there is a quasi-isomorphism as stated above. An explicit formula is given for example in the proof of [11, Lemma 7.2].

Let $A$ be a not necessarily unital $k$-algebra and write $\tilde{A}$ for its unitalization. Recall from [24] that $A$ is called $H$-unital if the groups $\operatorname{Tor}_{n}^{\tilde{A}}(k, A)$ vanish for all $n \geq 0$. Wodzicki proved in 24] that $A$ is $H$-unital if and only if for every embedding $A \triangleleft R$ of $A$ as a two-sided ideal of a unital ring $R$, the map

$$
H H(A) \rightarrow H H(R: A)=\operatorname{ker}(H H(R) \rightarrow H H(R / A))
$$

is a quasi-isomorphism.

Lemma 3.3. Theorem 3.1 still holds if the condition that $R$ be unital is replaced by the condition that it be $H$-unital.

Proof. Follows from Theorem 3.1 and the fact, proved in [11, Prop. A.6.5], that $R \rtimes G$ is $H$-unital if $R$ is as well.

Let $R$ be a unital algebra and $\phi: R \rightarrow p R p$ a corner isomorphism. As in [6], we consider the skew Laurent polynomial algebra $R\left[t_{+}, t_{-}, \phi\right]$. This is the $R$-algebra generated by elements $t_{+}$and $t_{-}$subject to the following relations:

$$
\begin{gathered}
t_{+} a=\phi(a) t_{+} \\
a t_{-}=t_{-} \phi(a) \\
t_{-} t_{+}=1 \\
t_{+} t_{-}=p
\end{gathered}
$$

Observe that the algebra $S=R\left[t_{+}, t_{-}, \phi\right]$ is $\mathbb{Z}$-graded by $\operatorname{deg}(r)=0, \operatorname{deg}\left(t_{ \pm}\right)= \pm 1$. The homogeneous component of degree $n$ is given by

$$
R\left[t_{+}, t_{-}, \phi\right]_{n}=\left\{\begin{array}{cc}
t_{-}^{-n} R & n<0 \\
R & n=0 \\
R t_{+}^{n} & n>0
\end{array}\right.
$$

Proposition 3.4. Let $R$ be a unital ring, $\phi: R \rightarrow p R p$ a corner isomorphism, and $S=R\left[t_{+}, t_{-}, \phi\right]$. Consider the weight decomposition $H H(S)=\bigoplus_{m \in \mathbb{Z}} H H(S)$. There is a quasi-isomorphism

$$
{ }_{m} H H(S) \stackrel{\sim}{\rightarrow} \text { Cone }\left(1-\phi: H H\left(R, S_{m}\right) \rightarrow H H\left(R, S_{m}\right)\right) .
$$

Proof. If $\phi$ is an automorphism, then $S=R \rtimes_{\phi} \mathbb{Z}$, the right hand side of (3.5) computes $\mathbb{H}\left(\mathbb{Z}, H H\left(R, S_{m}\right)\right)$, and the proposition becomes the particular case $G=$ $\mathbb{Z}$ of Theorem 3.1 In the general case, let $A$ be the colimit of the inductive system

$$
R \stackrel{\phi}{\longrightarrow} R \stackrel{\phi}{\longrightarrow} R \stackrel{\phi}{\longrightarrow} \ldots .
$$

Note that $\phi$ induces an automorphism $\hat{\phi}: A \rightarrow A$. Now $A$ is $H$-unital, since it is a filtering colimit of unital algebras, and thus the assertion of the proposition is true for the pair $(A, \hat{\phi})$, by Lemma 3.3. Hence it suffices to show that for $B=A \rtimes_{\hat{\phi}} \mathbb{Z}$ the maps $H H(S) \rightarrow H H(B)$ and Cone $\left(1-\phi: H H\left(R, S_{m}\right) \rightarrow H H\left(R, S_{m}\right)\right) \rightarrow$ Cone $\left(1-\phi: H H\left(A, B_{m}\right) \rightarrow H H\left(A, B_{m}\right)\right)(m \in \mathbb{Z})$ are quasi-isomorphisms. The analogous property for $K$-theory is shown in the course of the third step of the proof of [5, Thm. 3.6]. Since the proof in [5] uses only that $K$-theory commutes with filtering colimits and is matrix invariant on those rings for which it satisfies excision, it applies verbatim to Hochschild homology. This concludes the proof. 


\section{Hochschild homology of the Leavitt PATH AlgeBra}

Let $E=\left(E_{0}, E_{1}, r, s\right)$ be a finite quiver and let $\hat{E}=\left(E_{0}, E_{1} \sqcup E_{1}^{*}, r, s\right)$ be the double of $E$, which is the quiver obtained from $E$ by adding an arrow $\alpha^{*}$ for each arrow $\alpha \in E_{1}$, going in the opposite direction. The Leavitt path algebra of $E$ is the algebra $L(E)$ with one generator for each arrow $\alpha \in \hat{E}_{1}$ and one generator $p_{i}$ for each vertex $i \in E_{0}$, subject to the following relations:

$$
\begin{gathered}
p_{i} p_{j}=\delta_{i, j} p_{i} \quad\left(i, j \in E_{0}\right) \\
p_{s(\alpha)} \alpha=\alpha=\alpha p_{r(\alpha)} \quad\left(\alpha \in \hat{E}_{1}\right) \\
\alpha^{*} \beta=\delta_{\alpha, \beta} p_{r(\alpha)} \quad\left(\alpha, \beta \in E_{1}\right) \\
p_{i}=\sum_{\alpha \in E_{1}, s(\alpha)=i} \alpha \alpha^{*} \quad\left(i \in E_{0} \backslash \operatorname{Sink}(E)\right)
\end{gathered}
$$

The algebra $L=L(E)$ is equipped with a $\mathbb{Z}$-grading. The grading is determined by $|\alpha|=1,\left|\alpha^{*}\right|=-1$, for $\alpha \in E_{1}$. Let $L_{0, n}$ be the linear span of all elements of the form $\gamma \nu^{*}$, where $\gamma$ and $\nu$ are paths with $r(\gamma)=r(\nu)$ and $|\gamma|=|\nu|=n$. By [7, proof of Theorem 5.3], we have $L_{0}=\bigcup_{n=0}^{\infty} L_{0, n}$. For each $i$ in $E_{0}$ and each $n \in \mathbb{Z}^{+}$, let us denote by $P(n, i)$ the set of paths $\gamma$ in $E$ such that $|\gamma|=n$ and $r(\gamma)=i$. The algebra $L_{0,0}$ is isomorphic to $\prod_{i \in E_{0}} k$. In general, the algebra $L_{0, n}$ is isomorphic to

$$
\left[\prod_{m=0}^{n-1}\left(\prod_{i \in \operatorname{Sink}(E)} M_{|P(m, i)|}(k)\right)\right] \times\left[\prod_{i \in E_{0}} M_{|P(n, i)|}(k)\right] .
$$

The transition homomorphism $L_{0, n} \rightarrow L_{0, n+1}$ is the identity on the factors

$$
\prod_{i \in \operatorname{Sink}(E)} M_{|P(m, i)|}(k),
$$

for $0 \leq m \leq n-1$, and also on the factor

$$
\prod_{i \in \operatorname{Sink}(E)} M_{|P(n, i)|}(k)
$$

of the last term of the displayed formula. The transition homomorphism

$$
\prod_{i \in E_{0} \backslash \operatorname{Sink}(E)} M_{|P(n, i)|}(k) \rightarrow \prod_{i \in E_{0}} M_{|P(n+1, i)|}(k)
$$

is a block diagonal map induced by the following identification in $L(E)_{0}$ : A matrix unit in a factor $M_{|P(n, i)|}(k)$, where $i \in E_{0} \backslash \operatorname{Sink}(E)$, is a monomial of the form $\gamma \nu^{*}$, where $\gamma$ and $\nu$ are paths of length $n$ with $r(\gamma)=r(\nu)=i$. Since $i$ is not a sink, we can enlarge the paths $\gamma$ and $\nu$ using the edges that $i$ emits, obtaining paths of length $n+1$, and the last relation in the definition of $L(E)$ gives

$$
\gamma \nu^{*}=\sum_{\left\{\alpha \in E_{1} \mid s(\alpha)=i\right\}}(\gamma \alpha)(\nu \alpha)^{*} .
$$

Assume $E$ has no sources. For each $i \in E_{0}$, choose an arrow $\alpha_{i}$ such that $r\left(\alpha_{i}\right)=i$. Consider the elements

$$
t_{+}=\sum_{i \in E_{0}} \alpha_{i}, \quad t_{-}=t_{+}^{*}
$$


One checks that $t_{-} t_{+}=1$. Thus, since $\left|t_{ \pm}\right|= \pm 1$, the endomorphism

$$
\phi: L \rightarrow L, \quad \phi(x)=t_{+} x t_{-}
$$

is homogeneous of degree 0 with respect to the $\mathbb{Z}$-grading. In particular, it restricts to an endomorphism of $L_{0}$. By [6, Lemma 2.4], we have

$$
L=L_{0}\left[t_{+}, t_{-}, \phi\right] \text {. }
$$

Consider the matrix $N_{E}^{\prime}=\left[n_{i, j}\right] \in M_{e_{0}} \mathbb{Z}$ given by

$$
n_{i, j}=\#\left\{\alpha \in E_{1}: s(\alpha)=i, \quad r(\alpha)=j\right\} .
$$

Let $e_{0}^{\prime}=|\operatorname{Sink}(E)|$. We assume that $E_{0}$ is ordered so that the first $e_{0}^{\prime}$ elements of $E_{0}$ correspond to its sinks. Accordingly, the first $e_{0}^{\prime}$ rows of the matrix $N_{E}^{\prime}$ are 0 . Let $N_{E}$ be the matrix obtained by deleting these $e_{0}^{\prime}$ rows. The matrix that enters the computation of the Hochschild homology of the Leavitt path algebra is

$$
\left(\begin{array}{c}
0 \\
1_{e_{0}-e_{0}^{\prime}}
\end{array}\right)-N_{E}^{t}: \mathbb{Z}^{e_{0}-e_{0}^{\prime}} \longrightarrow \mathbb{Z}^{e_{0}}
$$

By a slight abuse of notation, we will write $1-N_{E}^{t}$ for this matrix. Note that $1-N_{E}^{t} \in M_{e_{0} \times\left(e_{0}-e_{0}^{\prime}\right)}(\mathbb{Z})$. Of course, $N_{E}=N_{E}^{\prime}$ in case $E$ has no sinks.

Theorem 4.4. Let $E$ be a finite quiver without sources, and let $N=N_{E}$. For each $i \in E_{0} \backslash \operatorname{Sink}(E)$ and $m \geq 1$, let $V_{i, m}$ be the vector space generated by all closed paths $c$ of length $m$ with $s(c)=r(c)=i$. Let $\mathbb{Z}=\langle\sigma\rangle$ act on

$$
V_{m}=\bigoplus_{i \in E_{0} \backslash \operatorname{Sink}(E)} V_{i, m}
$$

by rotation of closed paths. We have

$$
{ }_{m} H H_{n}(L(E))=\left\{\begin{array}{cc}
\operatorname{coker}\left(1-\sigma: V_{|m|} \rightarrow V_{|m|}\right) & n=0, m \neq 0 \\
\operatorname{coker}\left(1-N^{t}\right) & n=m=0 \\
\operatorname{ker}\left(1-\sigma: V_{|m|} \rightarrow V_{|m|}\right) & n=1, m \neq 0 \\
\operatorname{ker}\left(1-N^{t}\right) & n=1, m=0 \\
0 & n \notin\{0,1\}
\end{array}\right.
$$

Proof. Let $L=L(E), P=P(E) \subset L$ be the path algebras of $E$ and $W_{m} \subset P$ be the subspace generated by all paths of length $m$. For each fixed $n \geq 1$ and $m \in \mathbb{Z}$, consider the following $L_{0, n}$-bimodule:

$$
L_{m, n}=\left\{\begin{array}{cc}
L_{0, n} W_{m} L_{0, n} & m>0 \\
L_{0, n} W_{-m}^{*} L_{0, n} & m<0
\end{array}\right.
$$

Write $L=L(E)$, and let ${ }_{m} L$ be the homogeneous part of degree $m$; we have

$$
{ }_{m} L=\bigcup_{n \geq 1} L_{m, n}
$$

If $m$ is positive, then there is a basis of $L_{m, n}$ consisting of the products $\alpha \theta \beta^{*}$ where each of $\alpha, \beta$ and $\theta$ is a path in $E, r(\alpha)=s(\theta), r(\beta)=r(\theta),|\alpha|=|\beta|=n$ and $|\theta|=m$. Hence the formula

$$
\pi\left(\alpha \theta \beta^{*}\right)=\left\{\begin{array}{cc}
\theta & \text { if } \alpha=\beta \\
0 & \text { else }
\end{array}\right.
$$

defines a surjective linear map $L_{m, n} \rightarrow V_{m}$. One checks that $\pi$ induces an isomorphism

$$
H H_{0}\left(L_{0, n}, L_{m, n}\right) \cong V_{m} \quad(m>0) .
$$


Similarly, if $m<0$, then

$$
H H_{0}\left(L_{0, n}, L_{m, n}\right)=V_{|m|}^{*} \cong V_{-m} .
$$

Next, by (4.1), we have

$$
H H_{0}\left(L_{0, n}\right)=k[E \backslash \operatorname{Sink}(E)] \oplus \bigoplus_{i \in \operatorname{Sink}(E)} k^{r(i, n)} .
$$

Here

$$
r(i, n)=\max \{r \leq n: P(r, i) \neq \emptyset\} .
$$

Now note that because $L_{0, n}$ is a product of matrix algebras, it is separable, and thus $H H_{1}\left(L_{0, n}, M\right)=0$ for any bimodule $M$. As observed in (4.3), for the automorphism (4.2), we have $L=L_{0}\left[t_{+}, t_{-}, \phi\right]$. Hence in view of Proposition 3.4 and Lemma 2.1. it only remains to identify the maps $H H_{0}\left(L_{0, n}, L_{m, n}\right) \rightarrow H H_{0}\left(L_{0, n+1}, L_{m, n+1}\right)$ induced by inclusion and by the homomorphism $\phi$. One checks that for $m \neq 0$, these are respectively the cyclic permutation and the identity $V_{|m|} \rightarrow V_{|m|}$. The case $m=0$ is dealt with in the same way as in [5, Proof of Theorem 5.10].

Corollary 4.5. Let $E$ be a finite quiver with at least one non-trivial closed path.

i) $H H_{n}(L(E))=0$ for $n \notin\{0,1\}$.

ii) ${ }_{m} H H_{*}(L(E)) \cong{ }_{-m} H H_{*}(L(E))(m \in \mathbb{Z})$.

iii) There exist $m>0$ such that ${ }_{m} H H_{0}(L(E))$ and ${ }_{m} H H_{1}(L(E))$ are both nonzero.

Proof. We first reduce to the case where the graph does not have sources. By the proof of [5, Theorem 6.3], there is a finite complete subgraph $F$ of $E$ such that $F$ has no sources, $F$ contains all the non-trivial closed paths of $E, \operatorname{Sink}(F)=\operatorname{Sink}(E)$, and $L(F)$ is a full corner in $L(E)$ with respect to the homogeneous idempotent $\sum_{v \in F^{0}} p_{v}$. It follows that $H H_{*}(L(E))$ and $H H_{*}(L(F))$ are graded-isomorphic. Therefore we can assume that $E$ has no sources.

The first two assertions are already part of Theorem 4.4. For the last assertion, let $\alpha$ be a primitive closed path in $E$, and let $m=|\alpha|$. Let $\sigma$ be the cyclic permutation; then $\left\{\sigma^{i} \alpha: i=0, \ldots, m-1\right\}$ is a linearly independent set. Hence $N(\alpha)=\sum_{i=0}^{m-1} \sigma^{i} \alpha$ is a non-zero element of $V_{m}^{\sigma}={ }_{m} H H_{1}(L(E))$. Since on the other hand $N$ vanishes on the image of $1-\sigma: V_{m} \rightarrow V_{m}$, it also follows that the class of $\alpha$ in ${ }_{m} H H_{0}(L(E))$ is non-zero.

\section{Applications}

Theorem 5.1. Let $E_{1}, \ldots, E_{n}$ and $F_{1}, \ldots, F_{m}$ be finite quivers. Assume that $n \neq$ $m$ and that each of the $E_{i}$ and the $F_{j}$ has at least one non-trivial closed path. Then the algebras $L\left(E_{1}\right) \otimes \cdots \otimes L\left(E_{n}\right)$ and $L\left(F_{1}\right) \otimes \cdots \otimes L\left(F_{m}\right)$ are not Morita equivalent.

Proof. Immediate from Lemma 2.3 and Corollary 4.5(iii).

Example 5.2. It follows from Theorem 5.1 that $L_{2}$ and $L_{2} \otimes_{k} L_{2}$ are not Morita equivalent. There is another way of proving this, due to Jason Bell and George Bergman [8]. By Theorem 3.3 of [9], l.gl.dim $L_{2} \leq 1$. Using a module-theoretic construction, Bell and Bergman show that l.gl.dim $\left(L_{2} \otimes_{k} L_{2}\right) \geq 2$, which forces $L_{2}$ and $L_{2} \otimes_{k} L_{2}$ to not be Morita equivalent. Bergman then asked Warren Dicks whether general results were known about global dimensions of tensor products and was pointed to Proposition 10(2) of [12, which is an immediate consequence of 
Theorem XI.3.1 of [10] and says that if $k$ is a field and $R$ and $S$ are $k$-algebras, then l.gl.dim $R+$ w.gl.dim $S \leq$ l.gl.dim $\left(R \otimes_{k} S\right)$. Consequently, if l.gl.dim $R<\infty$ and w.gl.dim $S>0$, then l.gl.dim $R<$ l.gl.dim $\left(R \otimes_{k} S\right)$; in particular, $R$ and $R \otimes_{k} S$ are then not Morita equivalent. To see that w.gl.dim $L_{2}>0$, write $x_{1}, x_{2}, x_{1}^{*}$, $x_{2}^{*}$ for the usual generators of $L_{2}$ and use normal-form arguments to show that $\left\{a \in L_{2} \mid a x_{1}=a+1\right\}=\emptyset$ and $\left\{b \in L_{2} \mid x_{1} b=b\right\}=\{0\}$. Hence, in $L_{2}, x_{1}-1$ does not have a left inverse and is not a left zerodivisor (or see [4]); thus, w.gl.dim $L_{2}>0$.

We denote by $L_{\infty}$ the unital algebra presented by generators $x_{1}, x_{1}^{*}, x_{2}, x_{2}^{*}, \ldots$ and relations $x_{i}^{*} x_{j}=\delta_{i, j} 1$.

Proposition 5.3. Let $E$ be any finite quiver having at least one non-trivial closed path. Then $L_{\infty} \otimes L(E)$ and $L(E)$ are not Morita equivalent. Similarly, $L_{\infty} \otimes L_{\infty}$ and $L_{\infty}$ are not Morita equivalent.

Proof. Let $C_{n}$ be the algebra presented by generators $x_{1}, x_{1}^{*}, \ldots, x_{n}, x_{n}^{*}$ and relations $x_{i}^{*} x_{j}=\delta_{i, j} 1$, for $1 \leq i, j \leq n$. Then

$$
L_{\infty}=\underset{\lim }{\longrightarrow} C_{n}
$$

and $C_{n} \cong L\left(E_{n}\right)$, where $E_{n}$ is the graph having two vertices $v, w$ and $2 n$ arrows $e_{1}, \ldots, e_{n}, f_{1}, \ldots, f_{n}$, with $s\left(e_{i}\right)=r\left(e_{i}\right)=v=s\left(f_{i}\right)$ and $r\left(f_{i}\right)=w$ for $1 \leq i \leq n$. (The isomorphism $C_{n} \rightarrow L\left(E_{n}\right)$ is obtained by sending $x_{i}$ to $e_{i}+f_{i}$ and $x_{i}^{*}$ to $e_{i}^{*}+f_{i}^{*}$.) It follows from Theorem 4.4 and (5.4) that the formulas in Theorem 4.4 for ${ }_{m} H H_{n}\left(L_{\infty}\right), m \neq 0$, hold, taking as $V_{i, m}$ the vector space generated by all the words in $x_{1}, x_{2}, \ldots$ of length $m$, and that ${ }_{0} H H_{0}\left(L_{\infty}\right)=k$ and ${ }_{0} H H_{n}\left(L_{\infty}\right)=0$ for $n \geq 1$. As before, Lemma 2.3 gives the result.

Theorem 5.5. Let $E_{1}, \ldots, E_{n}$ and $F_{1}, \ldots, F_{m}, \ldots$ be a finite and an infinite sequence of quivers. Assume that the number of indices $i$ such that $F_{i}$ has at least one non-trivial closed path is infinite. Then the algebras $L\left(E_{1}\right) \otimes \cdots \otimes L\left(E_{n}\right)$ and $\bigotimes_{i=1}^{\infty} L\left(F_{i}\right)$ are not Morita equivalent.

Proof. Immediate from Lemma 2.3 and Corollary 4.5(iii).

Example 5.6. Let $L^{(\infty)}=\bigotimes_{i=1}^{\infty} L_{2}$, and let $E$ be any quiver having at least one non-trivial closed path. Then $L^{(\infty)} \otimes L(E)$ and $L(E)$ are not Morita equivalent.

It would be interesting to know the answer to the following question:

Question 5.7. Is there a unital homomorphism $\phi: L_{2} \otimes L_{2} \rightarrow L_{2}$ ?

Observe that to build a unital homomorphism $\phi: L_{2} \otimes L_{2} \rightarrow L_{2}$, it is enough to exhibit a non-zero homomorphism $\psi: L_{2} \otimes L_{2} \rightarrow L_{2}$ because $e L_{2} e \cong L_{2}$ for every non-zero idempotent $e$ in $L_{2}$.

\section{6. $K$-THEORY}

To conclude the paper we note that algebraic $K$-theory cannot distinguish between $L_{2}$ and $L_{2} \otimes L_{2}$ or between $L_{\infty}$ and $L_{\infty} \otimes L_{\infty}$. For this we need a lemma which might be of independent interest. Recall that a unital ring $R$ is said to be regular supercoherent in case all the polynomial rings $R\left[t_{1}, \ldots, t_{n}\right]$ are regular coherent in the sense of 13 . 
Lemma 6.1. Let $E$ be a finite graph. Then $L(E)$ is regular supercoherent.

Proof. Let $P(E)$ be the usual path algebra of $E$. It was observed in the proof of 3 , Lemma 7.4] that the algebra $P(E)[t]$ is regular coherent. The same proof gives that all the polynomial algebras $P(E)\left[t_{1}, \ldots, t_{n}\right]$ are regular coherent. This shows that $P(E)$ is regular supercoherent. By [3, Proposition 4.1], the universal localization $P(E) \rightarrow L(E)=\Sigma^{-1} P(E)$ is flat on the left. It follows that $L(E)$ is left regular supercoherent (see [5, page 23]). Since $L(E) \otimes k\left[t_{1}, \ldots, t_{n}\right]$ admits an involution, it follows that $L(E)$ is regular supercoherent.

Proposition 6.2. Let $R$ be regular supercoherent. Then the algebraic $K$-theories of $L_{2}$ and of $L_{2} \otimes R$ are both trivial.

Proof. Let $E$ be the quiver with one vertex and two arrows. Then $L_{2} \cong L(E)$, and we have

$$
L_{2} \otimes R=L_{R}(E) .
$$

Applying [5, Theorem 7.6] we obtain that $K_{*}\left(L_{R}(E)\right)=K_{*}(L(E))=0$. The result follows.

We finally obtain a $K$-absorbing result for Leavitt path algebras of finite graphs, indeed for any regular supercoherent algebra.

Proposition 6.3. Let $R$ be a regular supercoherent algebra. Then the natural inclusion $R \rightarrow R \otimes L_{\infty}$ induces an isomorphism $K_{i}(R) \rightarrow K_{i}\left(R \otimes L_{\infty}\right)$ for all $i \in \mathbb{Z}$.

Proof. Adopting the notation used in the proof of Proposition 5.3 we see that it is enough to show that the natural map $R \rightarrow R \otimes L\left(E_{n}\right)$ induces isomorphisms $K_{i}(R) \rightarrow K_{i}\left(R \otimes L\left(E_{n}\right)\right)$ for all $i \in \mathbb{Z}$ and all $n \geq 1$. Since $R$ is regular supercoherent, the $K$-theory of $R \otimes L\left(E_{n}\right) \cong L_{R}\left(E_{n}\right)$ can be computed by using [5, Theorem 7.6]. By the explicit form of the quiver $E_{n}$, we thus obtain that

$$
K_{i}\left(R \otimes L\left(E_{n}\right)\right) \cong\left(K_{i}(R) \oplus K_{i}(R)\right) /(-n, 1-n) K_{i}(R) .
$$

The natural map $R \rightarrow L_{R}\left(E_{n}\right)$ factors as

$$
R \rightarrow R v \oplus R w \rightarrow L_{R}\left(E_{n}\right) .
$$

The first map induces the diagonal homomorphism $K_{i}(R) \rightarrow K_{i}(R) \oplus K_{i}(R)$, sending $x$ to $(x, x)$. The second map induces the natural surjection

$$
K_{i}(R) \oplus K_{i}(R) \rightarrow\left(K_{i}(R) \oplus K_{i}(R)\right) /(-n, 1-n) K_{i}(R) .
$$

Therefore the natural homomorphism $R \rightarrow L_{R}\left(E_{n}\right)$ induces an isomorphism

$$
K_{i}(R) \stackrel{\sim}{\longrightarrow} K_{i}\left(L_{R}\left(E_{n}\right)\right) .
$$

This concludes the proof.

Corollary 6.4. The natural maps $k \rightarrow L_{\infty} \rightarrow L_{\infty} \otimes L_{\infty}$ induce $K$-theory isomorphisms $K_{*}(k)=K_{*}\left(L_{\infty}\right)=K_{*}\left(L_{\infty} \otimes L_{\infty}\right)$. 
Proof. A first application of Proposition 6.3 gives $K_{*}(k)=K_{*}\left(L_{\infty}\right)$. A second application shows that for $E_{n}$ as in the proof above, the inclusion $L\left(E_{n}\right) \rightarrow L\left(E_{n}\right) \otimes$ $L_{\infty}$ induces a $K$-theory isomorphism; passing to the limit, we obtain the corollary.

\section{ACKNOWLEDGEMENT}

Part of the research for this article was carried out during a visit of the second author to the Centre de Recerca Matemàtica. He is indebted to this institution for its hospitality.

\section{REFERENCES}

[1] G. Abrams, G. Aranda Pino. The Leavitt path algebra of a graph. J. Algebra 293 (2005) 319-334. MR2172342 (2007b:46085)

[2] G. Abrams, A. Louly, E. Pardo, C. Smith. Flow invariants in the classification of Leavitt path algebras. J. Algebra 333 (2011) 202-231. MR2785945

[3] P. Ara, M. Brustenga. Module theory over Leavitt path algebras and K-theory. J. Pure Appl. Algebra 214 (2010) 1131-1151. MR2586992 (2011b:16109)

[4] P. Ara, M. Brustenga. The regular algebra of a quiver. J. Algebra 309 (2007) 207-235. MR2301238(2008a:16019)

[5] P. Ara, M. Brustenga, G. Cortiñas. K-theory of Leavitt path algebras. Münster Journal of Mathematics 2 (2009) 5-34. MR2545605(2011d:46145)

[6] P. Ara, M.A. González-Barroso, K.R. Goodearl, E. Pardo. Fractional skew monoid rings. J. Algebra 278 (2004) 104-126. MR2068068 (2005f:16042)

[7] P. Ara, M. A. Moreno, E. Pardo, Nonstable K-theory for graph algebras. Algebr. Represent. Theory 10 (2007) 157-178. MR2310414 (2008b:46094)

[8] J. Bell, G. Bergman. Private communication, 2011.

[9] G. M. Bergman and Warren Dicks, Universal derivations and universal ring constructions. Pacific J. Math. 79 (1978) 293-337. MR531320 (81b:16024)

[10] H. Cartan, S. Eilenberg, Homological Algebra. Princeton University Press, Princeton, N. J., 1956. MR0077480 (17:1040e)

[11] G. Cortiñas, E. Ellis. Isomorphism conjectures with proper coefficients. arXiv:1108.5196v3.

[12] S. Eilenberg, A. Rosenberg, D. Zelinsky, On the dimension of modules and algebras, VIII. Dimension of tensor products. Nagoya Math. J. 12 (1957) 71-93. MR0098774 (20:5229)

[13] S. M. Gersten. K-theory of free rings. Comm. Algebra 1 (1974) 39-64. MR0396671 (53:533)

[14] E. Kirchberg, The classification of purely infinite $C^{*}$-algebras using Kasparov theory. Preprint.

[15] E. Kirchberg, N.C. Phillips, Embedding of exact $C^{*}$-algebras in the Cuntz algebra $\mathcal{O}_{2}$. J. Reine Angew. Math. 525 (2000) 17-53. MR.1780426 (2001d:46086a)

[16] M. Lorenz. On the homology of graded algebras. Comm. Algebra 20 (1992) 489-507. MR.1146311 (93b:19003)

[17] W. G. Leavitt. The module type of a ring. Trans. Amer. Math. Soc. 103 (1962) 113-130. MR 0132764(24:A2600)

[18] J. L. Loday, Cyclic homology. Grund. Math. Wiss., 301. Springer-Verlag, Berlin, Heidelberg, 1998. MR:1600246(98h:16014)

[19] N. C. Phillips, A classification theorem for nuclear purely infinite simple $C^{*}$-algebras. Doc. Math. 5 (2000) 49-114. MR1745197 (2001d:46086b)

[20] I. Raeburn. Graph algebras. CBMS Regional Conference Series in Mathematics, 103. Published for the Conference Board of the Mathematical Sciences, Washington, DC, by the American Mathematical Society, Providence, RI, 2005. MR.2135030 (2005k:46141)

[21] M. Rørdam, A short proof of Elliott's theorem. C. R. Math. Rep. Acad. Sci. Canada 16 (1994) 31-36. MR.1276341 (95d:46064)

[22] M. Rørdam, Classification of nuclear, simple $C^{*}$-algebras. Classification of nuclear $\mathrm{C}^{*}$ algebras. Entropy in operator algebras, Encyclopaedia Math. Sci., 126, 1-145, Springer, Berlin, 2002. MR,1878882(2003i:46060) 
[23] C. Weibel, An introduction to homological algebra, Cambridge Univ. Press, 1994. MR1269324 (95f:18001)

[24] M. Wodzicki, Excision in cyclic homology and in rational algebraic K-theory. Ann. of Math. 129 (1989) 591-639. MR997314 (91h:19008)

Departament de Matemàtiques, Universitat Autònoma de Barcelona, 08193 BellaTERra (BARCElona), Spain

E-mail address: para@mat.uab.cat

Departamento de Matemática and Instituto Santaló, Ciudad Universitaria Pab 1, 1428 Buenos Aires, Argentina

E-mail address: gcorti@dm.uba.ar

URL: http://mate.dm.uba.ar/ gcorti 